\title{
Implementation of the Common Vulnerability Scoring System to Assess the Cyber Vulnerability in Construction Projects
}

\author{
Bharadwaj Mantha ${ }^{1}$ and Yeojin Jung ${ }^{2}$ and Borja Garcia de Soto ${ }^{3}$
}

1 New York University Abu Dhabi (NYUAD), Abu Dhabi, United Arab Emirates, bmantha@nyu.edu

2 New York University Abu Dhabi (NYUAD), Abu Dhabi, United Arab Emirates, yj1254@nyu.edu

3 New York University Abu Dhabi (NYUAD), Abu Dhabi, United Arab Emirates,

garcia.de.soto@nyu.edu

\begin{abstract}
The utilization of new technologies coupled with the digitization and automation of the construction industry (known as Construction 4.0) comes with many advantages. For example, it will make the Architecture Engineering and Construction (AEC) industry more connected, accessible, and transparent. However, the inherent nature of these connected systems will make construction networks more vulnerable and prone to cyberattacks. That will compromise not only the confidentiality of sensitive information but also the security of physical assets and project participants. With this background in mind, it is crucial to measure the security of construction networks. There are different systems to evaluate security vulnerabilities of a system, network, organization, or process; one of the most common is the Common Vulnerability Scoring System (CVSS), which provides a numerical score that reflects the severity of a given vulnerability based on specific identified metrics. This paper examines the application of CVSS to quantify and evaluate the vulnerability of project participants that can be used as the groundwork to determine the security vulnerability of construction networks. The objectives of this paper are 1) to examine the advantages and disadvantages of different scoring systems and their applicability to the AEC industry, 2) to systematically apply the identified system to determine scores for some of the most significant construction participants such as the owner, contractor, and worker. The proposed approach will help to assess the vulnerability of project participants and, eventually, the security level of construction networks.
\end{abstract}

(c) 2020 The Authors. Published by Budapest University of Technology and Economics \& Diamond Congress Ltd Peer-review under responsibility of the Scientific Committee of the Creative Construction Conference 2020.

Keywords: construction automation, CVSS, cybersecurity, security score, vulnerability assessment, vulnerability metrics

\section{Introduction}

Construction project networks include the interaction between a wide range of participants, equipment, and assets. Such interaction often refers to the exchange of design specifics, work instructions, and information about the assets and participants of the project. The rapid rise of communication and information technologies has led the construction industry to progressively move towards the digital ecosystem, which is referred to as Construction 4.0. The aim is to have connected systems during all the phases of construction, starting from project inception till the end of life. This enables the utilization of data, which in many cases, contains highly confidential, sensitive, and proprietary information. This includes competitive bidding information, design specifications, engineered calculations, intellectual propertyrelated information, pricing, profit/loss data, banking records, employee information, quality, safety, and productivity-related standards and practices, to name a few. 
As has occurred in other industries, operating in a digital environment raises significant security concerns and vulnerable to cyberattacks. Within a computer system, a vulnerability is typically defined as a weakness that can be exploited by an attacker to perform unauthorized actions. In the context of this paper, vulnerability is referred to as a state or quality of being attacked or harmed because of a weakness in the system or user actions (e.g., weak software, unencrypted file sharing) [1]. Consequently, the actions performed by taking undue advantage of these vulnerabilities are referred to as attacks [2]. Most of the cyberattacks are either unreported because it causes damage to the reputation of the firm or unknown because the attacker uses sophisticated mechanisms to hide it [3]. A few examples of such reported cyberattacks concerning the construction industry include a) stolen design files of the highly sensitive Australian intelligence headquarter building [4]; b) attackers tried to steal proprietary related information of a robotic bricklayer [5]; c) an operational facility's (Target, a very well-known retailer in the United States) network was compromised, and attackers gained access to customers personal information through a vulnerable HVAC vendor who worked on the facility during the construction phase [6] and many more.

One of the fundamental ways to improve security is by quantifying the vulnerability of these organizational or project related networks [7]. Quantifying the network vulnerabilities enables identifying those that pose the highest risk, thereby assists in prioritizing and implementing network security solutions. By defining the security level of individual project participants and combining the individual scores into an overall measure for network security, we can identify the most vulnerable connections given a target participant and further suggest possible security solutions to improve the network. Some studies in the related fields like intelligent urban infrastructure systems, power grid systems, and water facilities proposed metrics to help quantify and assess the vulnerability of the respective networks (thereby the security of the network) [8] [9] [10]. However, these metrics are context and application-specific and do not apply to construction. For example, some of the context-specific metrics include the efficiency of the power grid, the power supply of the load stations, and the rate of pipe deterioration.

To summarize, none of the existing studies proposed a systematic approach for quantifying the security of individual participants and thereby the security of the entire construction network. This study serves as an initial step towards this direction. Specifically, the focus of this study is to a) explore different existing scoring systems and identify a suitable one for construction projects, and then b) systematically apply the scoring system to determine scores for some of the most significant construction participants such as the owner, contractor, or worker. The rest of this paper is organized as follows. Section 2 provides a review of the different scoring systems. In Section 3, the selected scoring system, namely the Common Vulnerability Scoring System (CVSS), is used in an example to determine the vulnerability score for different construction participants. Finally, Section 4 provides the conclusions and summarizes ongoing work by the authors in this area.

\section{Review of vulnerability scoring systems}

There are several existing vulnerability scoring systems managed by governmental, commercial, and nonprofit organizations. Each of these scoring systems focuses on different aspects of measuring vulnerability. For example, CERT/CC (Computer Emergency Response Team) considers whether the Internet infrastructure is at risk and establishes a procedure to examine the preconditions required to exploit the vulnerability [11]. The System Administration, Networking and Security (SANS) vulnerability analysis scale considers whether the weakness is found in default configurations or client or server systems [12]. Microsoft's proprietary scoring system reflects on the difficulty of exploitation and the overall impact of vulnerability [13]. While relevant and useful, these scoring systems assume that the impact of a vulnerability is constant for every individual and organization. This limits the usage of these systems for construction projects and networks which have different participants and varying security levels [14]. For example, vulnerability levels vary significantly between a contractor, worker, and owner. It is also possible that, depending on the type of the project (e.g., high-security government facility vs. residential project), the security level of these participants vary. Hence, it is necessary to quantify these based on customized networks rather than assigning constant values. 
Unlike the systems mentioned above, the Common Vulnerability Scoring System (CVSS) "provides a way to capture the principal characteristics of a vulnerability and produce a numerical score reflecting its severity" ${ }^{\prime \prime}$, or in other words, it assigns numerical scores to each identified vulnerability. This is a widely adopted system which is based on the relative security levels and, hence, enables security analysts, industry professionals, organizations, and researchers to adopt a common language in scoring the vulnerabilities. This is actively used by many organizations, including the United States (US) federal government systems and the National Institute of Standards and Technology (NIST). The US federal government uses this to rate the severity of vulnerabilities within their system [14]. The NIST National Vulnerability Database (NVD) provides a comprehensive database of CVSS vulnerability scores validated by the US government [14]. These scores enable organizations to understand and rank the impact of the vulnerabilities of individual subsystems and participants level.

Furthermore, there is a possibility to adjust the estimated scores and specifically tailor this scoring system to the target environment based on environmental metrics such as damage to the asset, revenue, or productivity. In addition, CVSS is also one of the six vulnerability management standards that comprise the Security Content Automation Protocol (SCAP). SCAP is a method to enable automated vulnerability management, measurement, and policy compliance evaluation (e.g., Federal Information Security Management Act ${ }^{2}$ (FISMA) compliance). U.S. government agencies and other organizations use SCAP to determine the presence of vulnerabilities and provide mechanisms to score the results of these measurements to evaluate the impact of discovered security issues. Therefore, considering all these advantages over other systems, in this study, the CVSS (version 3.1) [14] is systematically applied to different construction participants. Details regarding different elements of the CVSS, along with the quantitative evaluation of the score range is described in the following section.

\section{Applying CVSS to participants in construction projects}

The scores in the CVSS are estimated based on the three metrics, namely base metrics, temporal metrics, and environmental metrics. The base metrics capture the characteristics of the vulnerability that are consistent over a period of time and among different user environments. An example in the context of this study could be the vulnerability of construction entities due to the connected systems such as the Internet of Things and wireless networks on the job site. Optionally, the base score can be adjusted with temporal and environmental metrics to reflect time-variant and environment-specific factors, respectively. The temporal metric captures the readily available methods and techniques to exploit a vulnerability. A relevant example in construction could be an attacker remotely exploiting the vulnerability of a progress monitoring system (e.g., drone, camera) through a sophisticated attack (e.g., executing an automated agent software that allows the attacker to use target systems as attack platforms to perform malicious unauthorized actions). The objective of the environmental metrics is to understand the importance of the affected asset based on measuring the confidentiality, integrity, and availability. For example, in the context of construction, the environmental metric could relate to the effect of loss of confidentiality or integrity on the specific construction entity (e.g., contractor, worker, designer, and owner). Based on the examples mentioned above, it can be said that these three metrics are relevant to the AEC industry. However, for this study, only base metrics are considered. It has to be noted, however, that the same approach would apply for the inclusion of temporal and environmental metrics. For this study, it is assumed that the scores from the CVSS can be used to assess the severity of the vulnerability of the different participants in construction projects.

Acknowledging that the level of vulnerability varies depending on the construction participant (i.e., a small local contractor versus a large international contractor), this study uses a public owner (PO), a local contractor (LC), and a construction worker (CW) as the construction entities (i.e., participants) used to illustrate the implementation of the CVSS. This selection encompasses a varied range of security levels in the descending order assuming that a PO is expected to have a high amount of security (or conversely low

\footnotetext{
${ }^{1}$ Common Vulnerability Scoring System Special Interest Group (CVSS-SIG) [https://www.first.org/cvss/]

2 The act recognizes the importance of information security to the economic and national security interests of the United States.
} 
vulnerability) compared to that of an LC or a CW. The following sections describe the implementation of the CVSS to determine the vulnerability scores for each of the participants (or entities) indicated.

\subsection{Base metrics}

The score calculated using the base metrics is called the base score, which ranges between 0 and 10 . A higher base score refers to a higher exposure or higher vulnerability. Ranges from 0, (0.1 - 3.9), (4.0 - 6.9) (7.0 - 8.9), and (9.0 - 10) represent none, low, medium, high, and critical severity of vulnerability, respectively. There are three types of base metrics: exploitability, scope, and impact (as per the third version of the CVSS [14]). Each type is further divided into categories with different metric values and corresponding scores. Table 1 shows an overview of all the categories, metric values, and the corresponding score values for each of the three base metric types, as indicated in the CVSS guide. Each of these metric types, categories, and metric values are briefly defined as per the CVSS, and its relevance to the construction is illustrated with the help of the identified construction entities (PO, LC, and CW) previously introduced. For further in-depth details regarding each of the metric value definitions and scoring schema, the readers should refer to the CVSS guide [14].

Table 1. Scores for each of the base metric types, categories, and metric values according to the CVSS (adopted from [14])

\begin{tabular}{|c|c|c|c|c|c|c|c|}
\hline $\begin{array}{l}\text { Base } \\
\text { Metric } \\
\text { Type }\end{array}$ & Category & Metric Value & Score & $\begin{array}{l}\text { Base } \\
\text { Metric } \\
\text { Type }\end{array}$ & Category & Metric Value & Score \\
\hline \multirow{11}{*}{$\begin{array}{l}\text { Exploitability } \\
\text { Metrics }\end{array}$} & \multirow{4}{*}{$\begin{array}{l}\text { Access } \\
\text { Vector (AV) }\end{array}$} & Physical $(\mathrm{P})$ & 0.20 & \multirow{2}{*}{$\begin{array}{l}\text { Scope } \\
\text { (S) }\end{array}$} & \multirow[b]{2}{*}{ - } & Unchanged $(U)$ & - \\
\hline & & Local (L) & 0.55 & & & Changed $(\mathrm{C})$ & - \\
\hline & & Adjacent Network (A) & 0.62 & \multirow{9}{*}{$\begin{array}{l}\text { Impact } \\
\text { Metrics }\end{array}$} & \multirow{3}{*}{$\begin{array}{l}\text { Confidentiality } \\
\text { (C) }\end{array}$} & None $(\mathrm{N})$ & 0.00 \\
\hline & & Network $(\mathrm{N})$ & 0.85 & & & Low (L) & 0.22 \\
\hline & \multirow{2}{*}{$\begin{array}{l}\text { Attack } \\
\text { Complexity } \\
\text { (AC) }\end{array}$} & $\operatorname{High}(\mathrm{H})$ & 0.44 & & & $\operatorname{High}(\mathrm{H})$ & 0.56 \\
\hline & & Low (L) & 0.77 & & \multirow{3}{*}{ Integrity (I) } & None (N) & 0.00 \\
\hline & \multirow{3}{*}{$\begin{array}{l}\text { Privileges } \\
\text { Required } \\
\text { (PR) }\end{array}$} & High $(\mathrm{H})$ & $\begin{array}{l}0.27^{*} \\
\text { or } \\
0.50^{\star \star}\end{array}$ & & & Low (L) & 0.22 \\
\hline & & Low (L) & $\begin{array}{l}0.62^{*} \\
\text { or } \\
0.68^{* *}\end{array}$ & & & High $(H)$ & 0.56 \\
\hline & & None (N) & 0.85 & & \multirow{3}{*}{ Availability (A) } & None $(\mathrm{N})$ & 0.00 \\
\hline & \multirow{2}{*}{$\begin{array}{l}\text { User } \\
\text { Interaction } \\
\text { (UI) }\end{array}$} & Required (R) & 0.62 & & & Low $(\mathrm{L})$ & 0.22 \\
\hline & & None (N) & 0.85 & & & High $(\mathrm{H})$ & 0.56 \\
\hline
\end{tabular}

Note: *for Scope = Unchanged (U); **for Scope $=$ Changed $(C)$

\subsubsection{Exploitability metrics}

The objective of this metric is to measure the relative difficulty of exploiting a particular vulnerability. In the current context, it is to measure the vulnerability of a particular construction entity that could lead to a successful attack. The following paragraphs go over the four categories that comprise the exploitability metrics.

a) Access Vector (AV) category measures the distance accessibility required for the hacker to exploit a vulnerability. That is, how far the attacker can be and still manage to attack the entity. For example, in the case of construction, does the attacker need to be physically present on the job site? Can the attacker launch an attack on the autonomous equipment remotely? The more remote an attacker can be to launch an attack, the more vulnerable the entity is, and hence the higher the vulnerability score of the entity. The AV levels are divided into four metric values, namely Physical (P) access (e.g., physically access the target system), Local (L) access (e.g., local account required or intended or unintended user interaction such as trick a user to open a malicious document), Adjacent Network (A) access (e.g., accessible from local subnetworks), and Network (N) access (e.g., accessible through the Internet). For example, to attack a sophisticated entity like a high profile PO, an attacker needs to have physical access (i.e., Metric Value = 'Physical' and Score $=0.20$ from Table 1), considering that the PO has stringent cybersecurity policies and procedures in place. However, the attacker could potentially attack a CW's mobile phone or any device where work-related information is stored remotely through the internet (i.e., Metric Value $=$ 'Network' and Score $=0.85$ from Table 1 ). It is thus reasonable to assume that an average LC lies in the middle of the 
spectrum (i.e., between 'Physical' and 'Network'), and hence local access is considered (i.e., Metric Value = 'Local' and Score $=0.55$ from Table 1).

b) Attack Complexity (AC) category measures the complexity of the attack required to exploit the vulnerability once the access is made. That is, how complex should the attack be to compromise any specific construction entity. For example, a Common Data Environment (CDE) managed and controlled by a contractor (or designer) is considered highly vulnerable if all the users (e.g., painting subcontractor) have complete access and rights to the structural, mechanical, electrical, and plumbing shop drawings. Possible metric values, as suggested by the CVSS, include High (H) (e.g., admin/root privilege required) and Low (L) (e.g., no specialized access condition required). As can be seen from the corresponding scores in Table 1, the lower the required complexity, the higher the vulnerability score. For example, the attacker would need an admin or root privilege to access or modify any of the information if he/she gets access to any of the PO's network. Hence a value of 'High' is considered for the PO (Score $=0.44$ from Table 1), and correspondingly, 'Low' (Score $=0.77$ from Table 1 ) is considered for both the LC and CW.

c) Privileges Required (PR) category relates to the level of exclusive rights an attacker is required to possess to exploit a vulnerability. In the context of this study, that means how much privilege will an attacker require to compromise any construction entity's network (e.g., PO's subnetwork), equipment (e.g., CW's tablet interface), or repository (LC's CDE repository). Possible CVSS metric values include High (e.g., administrative control required), Low (e.g., user control required to impact the settings and files owned by the user), and None (e.g., does not require any prior access but still can launch an attack). Considering that a PO will have multi-layer security, high privileges would be required to hack his/her systems, components, or networks. That is why 'High' is chosen for the PO, and correspondingly a score of 0.27 (if Scope $=$ Unchanged) or 0.50 (if Scope $=$ Changed) is assigned depending on the Scope (which is described later in this section of the paper). Furthermore, it is reasonable to assume a 'Low' type for both the LC and CW. This is because of the bare minimum security every entity has, starting from the usage of their mobile phones to work laptops or tablets. Therefore, a score of 0.62 (if Scope $=$ Unchanged) or 0.68 (if Scope $=$ Changed), depending on the Scope, is assigned for those participants.

d) User Interaction (UI) category captures the requirement of an internal or external user, other than the attacker, to assist in successfully launching an attack on the vulnerable component. In the current context, it relates to an external or an internal user (e.g., existing employee) assisting a potential attacker in compromising any construction entity. This category is further divided into two metric values: Required (R) and None (N). For example, to successfully exploit a PO, an attacker might need the help of a network administrator. However, none of such user interaction might be required to launch a similar attack on the LC and CW considering the technology readiness of these entities. For instance, an attacker might not need administrative privileges to be able to hack an LC or CW's personal device, which might have been frequently used to access work-related information. Hence values of 'Required' (Score $=0.62$ from Table 1) were assigned to the PO and 'None' (Score $=0.85$ from Table 1 ) to the LC and CW.

\subsubsection{Scope (S)}

This metric captures whether a component's vulnerability can impact resources and components beyond its scope. In the context of this study, it is to capture whether the vulnerability of a specific construction entity can impact components such as the assets, information, and resources of other entities. This metric type has two metric values: Unchanged $(U)$ and Changed (C). As the name suggests, a metric value of ' $U$ ' suggests that there will be no change in scope, and conversely, a value of ' $C$ ' means that there will be a change in scope. Due to the inherent nature of construction, almost all the entities are interrelated and hence can compromise and impact each other with very few exceptions. For example, [15] showed how the vulnerability of an individual entity impacts the whole construction network due to the existing information and communication exchange channels. An example of an exception can be when a potential vendor who is not part of the construction project yet, might not have any communication or association with the existing entities (e.g., contractor), and hence the vulnerability of such a potential entity might not have any impact. In the context of this study, all the participants considered can either have a major or minor impact on the other entities. Hence a metric value of Changed (C) is assigned for the PO, LC, and CW. As briefly 
discussed in 3.1.1 c), the score for some of the values for the Privilege Required (PR) category varies depending on the metric value used for the Scope. Since we are assuming a 'Changed' metric value for Scope, the metric values for the PR category corresponding to the Changed condition should be used. Therefore, the PR values, in this case, are 0.50 for PO and 0.68 for LC and CW.

\subsubsection{Impact metrics}

The idea of the impact metric is to capture the effect of exploiting a vulnerability. That is, in the current context, measure the impact an attacker can have on the specific construction entity if an attack is successfully launched. As can be expected, the increased impact increases the vulnerability score. The following paragraphs go over the three categories that comprise the impact metrics.

a) Confidentiality Impact (C) category measures the impact on information confidentiality. That is, to measure the impact of disclosing or revealing the information of a construction entity. For example, an attacker steals the private encryption key of a contractor's or a CDE's server. This could lead to gaining access to the design files, contractual documents, and sensitive personal records of the employees, visitors, or workers. Possible metric values are 'None' (e.g., no impact), 'Low' (e.g., considerable disclosure of information), and 'High' (e.g., total disclosure). Considering the three participants, it is reasonable to assume that, even though the attacker somehow accesses the PO's systems, he/she might have a 'Low' confidential impact (i.e., Score $=0.22$ from Table 1 ) because of the possible defense mechanisms that might already be in place. However, a similar attack on the LC or CW will have a 'High' impact (i.e., Score $=0.56$ from Table 1).

b) Integrity Impact (I) category measures the ability of the attacker to modify, tamper, or delete an entity's information. In the context of this study, it is to measure the extent of modification an attacker can perform once the construction entity is hacked. Considering a similar example as in the Confidentiality Impact above, the attacker not only has access to the restricted information related to the design, contract, construction, and operation but also can modify or erase it. Possible metric values are 'None' (e.g., no impact), 'Low' (e.g., modification of some information), and 'High' (e.g., complete loss of system protection). Similarly, a value of 'Low' (i.e., Score $=0.22$ from Table 1 ) is considered for the PO, and a value of 'High' (i.e., Score $=0.56$ from Table 1) is used for both the LC and the CW.

c) Availability Impact (A) category measures the ability of the attacker to impact the availability of the target system. In the context of this study, it measures the amount of control an attacker can have once the specific entity or the entity's resources. Possible metric values are None (e.g., no impact), Low (e.g., reduced performance or interruptions in resource availability), and High (e.g., total shutdown of the affected resource). For example, if an attacker hacks the tower crane on site, which is usually controlled by the LC, it is possible to take over that resource and render it completely dysfunctional. Considering the cyber awareness and readiness of the CW, it could also happen the same for any of the resources he/she might be operating. Therefore, a metric value of High is considered for both these participants, and a score of 0.56 is assigned. However, a PO might have an existing defense mechanism in place that might usually block a hacker from doing so and might end up with some loss of functionality. Hence, a value of low (i.e., Score $=0.22$ from Table 1 ) is considered.

All the scores for the three construction entities (PO, LC, and CW) and the different categories are summarized in Table 2.

\subsubsection{Base score calculation}

As shown in Table 1, each of the metric values is mapped to the corresponding numerical score. With the help of these scores, a CVSS base score can be calculated using equations 1 to 4 from the CVSS guide [14]. While equations 2,3 , and 4 help estimate individual base metric type scores, equation 1 aggregates all these to estimate the final base score. The corresponding values calculated for the PO, LC, and CW are shown in Table 2. As can be seen, the PO, LC, and CW have total base scores of 4.28, 8.71, and 9.89, respectively. Those scores represent a medium, high, and critical vulnerability, respectively, based on the 0 to 10 score range mentioned at the beginning of this section. 
BaseScore $=0$, if (Impact Score $\leq 0$ ), else

$$
\begin{aligned}
& =\text { Roundup }\{\text { Minimum }[(\text { Impact Score }+ \text { Exploitability Score }), 10] \text { if }(\text { Scope }=\text { Unchanged }) \\
& =\text { Roundup }\{\text { Minimum }[1.08 *(\text { Impact Score }+ \text { Exploitability Score }), 10] \text { if }(\text { Scope }=\text { Changed })
\end{aligned}
$$

Exploitability Score $=8.22 *$ AccessVector $*$ AttackComplexity $*$ PrivilegesRequired $*$ UserInteraction

Impact Sub Score $(I S S)=(1-(1-$ Confidentiality $) *(1-$ Integrity $) *(1-$ Availability $))$

Impact Score $=6.42 *$ ISS if $($ Scope $=$ Unchanged $)$

$$
=7.52 *(I S S-0.029)-3.25 *(I S S-0.02)^{15} \text { if }(\text { Scope }=\text { Changed })
$$

\begin{tabular}{|c|c|c|c|c|c|c|c|}
\hline \multirow{2}{*}{$\begin{array}{l}\text { Base } \\
\text { Metrics }\end{array}$} & \multirow{2}{*}{ Category } & \multicolumn{2}{|c|}{$\begin{array}{l}\text { Public } \\
\text { Owner (PO) }\end{array}$} & \multicolumn{2}{|c|}{$\begin{array}{l}\text { Local } \\
\text { Contractor (LC) }\end{array}$} & \multicolumn{2}{|c|}{$\begin{array}{l}\text { Construction } \\
\text { Worker (CW) }\end{array}$} \\
\hline & & $\begin{array}{l}\text { Metric } \\
\text { Value }\end{array}$ & Score & $\begin{array}{l}\text { Metric } \\
\text { Value }\end{array}$ & Score & $\begin{array}{l}\text { Metric } \\
\text { Value }\end{array}$ & Score \\
\hline \multirow{4}{*}{ Exploitability } & AV & $\mathrm{P}$ & 0.20 & L & 0.55 & $\mathrm{~N}$ & 0.85 \\
\hline & $A C$ & $\mathrm{H}$ & 0.44 & L & 0.77 & L & 0.77 \\
\hline & PR & $\mathrm{H}$ & 0.50 & $\mathrm{~L}$ & 0.68 & L & 0.68 \\
\hline & UI & $\mathrm{R}$ & 0.62 & $\mathrm{~N}$ & 0.85 & $\mathrm{~N}$ & 0.85 \\
\hline Scope & - & $\mathrm{C}$ & - & $\mathrm{C}$ & - & $\mathrm{C}$ & - \\
\hline \multirow{3}{*}{ Impact } & $\mathrm{C}$ & L & 0.22 & $\mathrm{H}$ & 0.56 & $\mathrm{H}$ & 0.56 \\
\hline & 1 & L & 0.22 & $\mathrm{H}$ & 0.56 & $\mathrm{H}$ & 0.56 \\
\hline & $A$ & L & 0.22 & $\mathrm{H}$ & 0.56 & $\mathrm{H}$ & 0.56 \\
\hline \multicolumn{2}{|c|}{ Exploitability Score (Eq. 2) } & & 0.22 & & 2.01 & & 3.11 \\
\hline \multicolumn{2}{|c|}{ Impact Sub Score (ISS) (Eq. 3) } & & 0.53 & & 0.91 & & 0.91 \\
\hline \multicolumn{2}{|c|}{ Impact Score (Eq. 4) } & & 3.73 & & 6.05 & & 6.05 \\
\hline \multicolumn{2}{|c|}{ Total Base Score (Eq. 1) } & & 4.28 & & 8.71 & & 9.89 \\
\hline
\end{tabular}

Table 2. Base metric score calculations for each of the construction entities considered

\section{Conclusion and ongoing work}

Construction is progressively moving towards adopting automation and digitalization technologies. This will inevitably increase the number of cyberattacks that occur in the industry. Based on the amount of available scientific literature, it seems that the issue of construction cybersecurity is at a very nascent stage. Though several standards and policies exist for risk assessment and mitigation in the construction project management domain, a widely accepted cybersecurity assessment is mostly unavailable in the construction. One of the fundamental steps towards analyzing and addressing the cybersecurity concerns include the ability to quantitatively measure and understand the security, or conversely, the vulnerability of participants (e.g., owners, architects, and contractors) and networks (e.g., combined group of participants, equipment, and assets). This study provides an impetus towards research in this direction and explores different systems that exist in the cybersecurity domain. Preliminary analysis of different available systems suggests that the Common Vulnerability Scoring System (CVSS) has several advantages compared to that of others and allows customizable scores for different entities.

The CVSS was systematically applied to determine the total base scores for three of the construction participants, namely a public owner (PO), a local contractor (LC), and a construction worker (CW). For the sake of simplicity, only the base metric scoring was shown in this study. This could, however, be extended to adjust the base scores and incorporate the temporal and environmental metrics. Based on the ranges of the base scores defined by CVSS, the PO, LC, and CW had a medium, high, and critical vulnerability. These scores need not necessarily be an exact representation of all the owners, contractors, or workers, and caution should be exercised to avoid generalizing the results from this study. Instead, this study should be used as an example to implement the CVSS to determine the vulnerability level of each of the entities by considering the individual characteristics of the entity (or participant) in a construction project. Further investigation, such as comparing attack history and assessing the security of different systems within the network or subnetwork, can be done to assign more precise metric values. 
Ongoing work of the authors extends this scoring system to define and analyze the overall vulnerability of construction networks. This is done by adopting the CVSS aggregation approaches suggested in the literature. Such an analysis could greatly assist the existing risk assessment (e.g., comparing the cyber risks of different project delivery methods or supply chain structures) and management approaches (e.g., investigating the cyber vulnerabilities of introducing drones or 3D printers on construction sites).

\section{Acknowledgment}

The authors would like to thank the support from the Center for Cyber Security at New York University Abu Dhabi (NYUAD).

\section{References}

[1] M. J. Hutchins, R. Bhinge, M. K. Micali, S. L. Robinson, J. W. Sutherland, and D. Dornfeld, "Framework for Identifying Cybersecurity Risks in Manufacturing," Procedia Manufacturing, vol. 1, pp. 47-63, Jan. 2015, doi: https://doi.org/10.1016/j.promfg.2015.09.060.

[2] M. Abomhara and G. M. Koien, "Cyber Security and the Internet of Things: Vulnerabilities, Threats, Intruders and Attacks," Journal of Cyber Security and Mobility, vol. 4, no. 1, pp. 65-88, Jan. 2015, doi: https://doi.org/10.13052/jcsm2245-1439.414.

[3] I. T. R. C. ITRC, "END-OF-YEAR DATA BREACH," 2019. [Online]. Available: https://www.idtheftcenter.org/wpcontent/uploads/2019/02/ITRC_2018-End-of-Year-Aftermath_FINAL_V2_combinedWEB.pdf. [Accessed: 16-Mar-2020].

[4] S. Watson, "Cyber-security: What will it take for construction to act? | Construction News," 2018. [Online]. Available: https://www.constructionnews.co.uk/tech/cyber-security-what-will-it-take-for-construction-to-act-22-01-2018/. [Accessed: 16-Mar2020].

[5] C. Pash, "How hackers and spies tried to steal the secrets of Australia's one-armed robot bricklayer | Business Insider," 2018. [Online]. Available: https://www.businessinsider.com.au/one-armed-bricklaying-robot-security-secrets-2018-11. [Accessed: 16-Mar2020].

[6] X. Shu, K. Tian, A. Ciambrone, and D. Yao, "Breaking the Target: An Analysis of Target Data Breach and Lessons Learned," Cornell University; Computer Science, Cryptography and Security, Jan. 2017.

[7] P. Cheng, L. Wang, S. Jajodia, and A. Singhal, "Aggregating CVSS Base Scores for Semantics-Rich Network Security Metrics," in IEEE 31st Symposium on Reliable Distributed Systems, 2012, pp. 31-40, https://doi.org/10.1109/SRDS.2012.4.

[8] A. Agathokleous, C. Christodoulou, and S. E. Christodoulou, "Robustness and vulnerability assessment of water networks by use of centrality metrics," European Water Resources Association, vol. 58, pp. 489-495, 2017.

[9] M. Ouyang, L. Hong, Z. Pan, and L. Zhao, "Correlation analysis of different vulnerability metrics on power grids Resilience Optimization of Interdependent Critical Infrastructures View project Correlation analysis of different vulnerability metrics on power grids," Physica A, vol. 396, pp. 204-211, 2014, doi: https://doi.org/10.1016/j.physa.2013.10.041.

[10] J. L. Bayuk and A. Mostashari, "Measuring Cyber Security in Intelligent Urban Infrastructure Systems," in 8th International Conference \& Expo on Emerging Technologies for a Smarter World, 2011, pp. 1-6, https://doi.org/10.1109/CEWIT.2011.6135873.

[11] U. S. C. E. R. T. US-CERT, "CERT Vulnerability Notes Database," CERT/CC Vulnerability Notes Database, 2006. [Online]. Available: https://kb.cert.org/vuls/bypublished/desc/. [Accessed: 16-Mar-2020].

[12] S. Cima, "SANS Institute Information Security Reading Room - Vulnerability Assessment," SANS Institute, 2001. [Online]. Available: https://www.sans.org/reading-room/whitepapers/basics/vulnerability-assessment-421. [Accessed: 16-Mar-2020].

[13] C. Microsoft, "Security Update Severity Rating System," Microsoft Security Response Center Security Bulletin Severity Rating System., 2007. [Online]. Available: https://www.microsoft.com/en-us/msrc/security-update-severity-rating-system. [Accessed: 16-Mar2020].

[14] FIRST, "Common Vulnerability Scoring System version 3.1: Specification Document," 2019. [Online]. Available: https://www.first.org/cvss/v3-1/cvss-v31-specification_r1.pdf. [Accessed: 25-Mar-2020].

[15] B. R. K. Mantha and B. Garcia de Soto, "Cyber security challenges and vulnerability assessment in the construction industry," in Proceedings of the Seventh Creative Construction Conference (CCC 2019), 29 June - 2 July 2019, Budapest, Hungary., p. 9, doi: https://doi.org/10.3311/CCC2019-005. 